proyectos que Bellido tenia como propios del Instituto (luego, es cierto, han venido otros y hemos ganado otros); no sé si hemos perdido el archivo fotográfico. Hemos estado a punto de perder Archivo, lo que hubiera significado acabar con el Instituto, perderlo todo.

No es mi tarea aqui decir qué es lo que se debe hacer en el futuro; mi tarea ha sido recordar cómo nació y cuáles eran los fundamentos y las ideas guia con las que se creó el Dpto. de Arqueologia del ('SIC. Un Dpto. que el año 2001 cumplirá 50 años de historia, que habrá de celebrar adecuadamente, porque no debemos renunciar a la historia, una historia esforzada de todos sus muchos colaboradores, una historia, que a pesar de todo, creo que hace honor a su fundador, una historia de compromiso con la investigación de alta calidad y rigor que es la que practican cotidianamente sus miembros de todas las escalas.

\title{
LA URBANÍSTICA DEL MUNDO IBÉRICO. LAS NUEVAS PERSPECTIVAS
}

POR

\author{
L. ABAD y M. BENDALA \\ Universidad Autónoma de Madrid
}

Hace cincuenta anıs, el conocimiento que se tenía de la urbanística y la arquitectura ibérica era muy limitado y fragmentario. En palabras de D. Antonio García y Bellido, según escribia en los años cuarenta, «el número y nombre de las ciudades ibéricas conocidas por los textos antiguos era grande, sobre todo en Andalucia y Levante; pero desgraciadamente son poquísimas las que han llegado a nosotros aun en ruinas, y éstas son siempre, o casi siempre, ciudades que por su área, sus construcciones y el mismo anonimato con que se han dado a la luz, denuncian haber sido de rango relativamente bajo") (Ars Hispaniae I, Madrid, 1947, 203). Es la idea, expresada en un párrafo muy sintético, que desarrolla en trabajos de la época y algo posteriores (La Arquitectura entre los iberos, Madrid, 1945; Historia de España de R. M. Pidal, 1-3, Madrid, 1954, 373ss.), y corroboran los demás autores, entre ellos A. Arribas, en su conocida sintesis sobre Los Iberos, de unos años después (Barcelona, 1965): «Por desgracia, el conocimiento que tenemos de los núcleos de población ibéricos es muy limitado» (p. 117).

Era evidente un conocimiento muy parcial, y particularmente escaso o nulo para la zona de mediodía, la que según todos los indicios habia conocido un mayor desarrollo del urbanismo y el florecimiento de las ciudades de mayor rango. Se tenía en ello una consecuencia de la diferente intensidad de la investigación arqueológica en unas zonas y otras $y$, además, el resultado del diverso grado de continuidad histórica de los centros urbanos desde la Antiguedad a nuestros dias.

En cualquier caso, el despegue económico y cientifico a partir de los sesenta, que ha conducido a la multiplicación de los equipos cientificos y de las excavaciones, y la aplicación de una politica patrimonial más respetuosa con el legado arqueológico, permiten situarnos hoy ante un panorama completamente nuevo a la hora de contemplar el urbanismo ibérico.

El cambio cuantitativo (muchos más datos) y cualitativo (mejorregistro arqueológico, nuevos planteamientos metodológicos y teóricos) puede resumirse y plantearse en función de los dos planos principales en que se proyecta la ciudad; el conjunto de la organización urbana y las formas urbanisticas concretas de cada núcleo o tipo de centros. Dicho en otras palabras: lo urbano y lo urbanístico.

Se ha intensificado la investigación en busca de una aclaración definitiva a la cuestión de si el mundo ibérico alcanzó o no niveles urbanos. El resultado, con discusiones inevitables, es que el mundo ibérico es una cultura de nivel urbano, y la pregunta, en cada caso, no puede o no debe plantearse - a la altura de nuestro tiempo-en el sentido de si éste o aquel centro es una ciudad, sino si pertenece a una organización urbana y qué lugar y papel ocupa en ella. Se reconoce en el mundo ibérico una estruc- 
tura j erarquizada y complej a. que no sólo permite la existencia de diferentes tipos de centro, sino que los exige en virtud de determinadas formas de control y de explotación de la tierra, y de contactos con ambitos territoriales cada vę más amplios. El estudie de la dimensión territorial de la ciudad, con un fecundo diálogo, en cuestiones metodológicas, entre los resultados de exeavaciones puntuales y de prospecciones sistematicas de amplio aleance. configura uno de los campos más avanzados y atractivos en el conocimiento de la cultura ibérica. La reconstrucción del paisaje natural y antrópico de la época es un objetivo prioritario, en el que se van obteniendo logros considerables.

El progreso en la definición de la estructura urbana se ha ido consolidando en sus dos vertientes definitorias principales: la espacial y la cronológica. Para lo uno y lo otro, la referencia a la base tartésica resulta imprescindible. Sólo con decir esto se subrayan tanto la antiguedad del urbanismo ibérico como el reconocimiento de una región de vanguardia en la zona nuclear tartésica. Excavaciones en numerosos yacimientos (Mesa de Asta, Carambolo Carmona, Macareno, Huelva, Tejada la Vieja, Montemolin, Setefilla, Córdoba, Montoro, etc.), acreditan ese fenómeno. Su irradiación cultural y su consolidación por el contacto reciente con los colonos mediterráneos pondrá las bases de desarrollos regionales en los que se inserta lo que se entiende como cultura ibérica clásica. En ésta, los datos de su madurez urbana y urbanística remontan ya con seguridad al siglo VI y los comienzos del v, lo que acreditan los núcleos de hábitat excavados (entre ellos EI oral), las necrópolis y, muy expresivamente, la producción artística, con niveles y facetas, sobre todo en la escultura mayor, que sólo se explican en contextos urbanos (sociológicos, económicos, culturales) desarrollados. Con diferentes vicisitudes, esta organización urbana será la base que haga posible la conquista romana y la potenciación y ampliación de su estructura como consecuencia de la romanización, otra de las facetas tratadas intensamente en la investigación reciente.

Geográficamente, el mediodia es escenario de un gran desarrollo urbano, reflejado también en la existencia de las mayores aglomeraciones ciudadanas (Carmo, Carteia, Castulo, Carthago Nova, etc.). El módulo se reduce en las culturas de levante $y$ aún más en zonas de influencia en los ámbitos celtibéricos (como se ve en los abundantes yacimientos conocidos del valle del Ebro). En cualquier caso, el reconocimiento de una irradiación de la cultura ibérica hacia el interior peninsular cada vez más profunda e intensa, es otra de las consecuencias de la investigación reciente. reveladora de interesantes fenómenos de relación centro-periferia.

La comprensión de la organización urbana va ganando terreno igualmente en aspectos como la red viaria, el reconocimiento de contactos zonales privilegiados, la valoración del papel de centros no urbanos, como los santuarios, en la integración de diferentes ciudades. En esto, la historiogratia tradicional forjó una imagen de la ciudad ibérica en la que era una nota peculiar la ausencia de centros de culto, que se ubicaban por lo general fuera, en parajes naturales. Es éste otro aspecto desmentido por la investigación moderna, que equipara las aglomeraciones ibéricas a las de tantas otras culturas en las que el templo es un referente urbano de primera magnitud. Los casos de la Escuera, la Alcudia de Elche, la Isleta de Campello, Torreparedones, el Amarejo, San Miguel de Liria, Ullastret, Azaila y otros, revelan este hecho, tanto gracias a excavaciones modernas como a la revisión con nuevos presupuestos de las antiguas (otra labor acometida con importantisimos resultados).

En los apartados urbanisticos de las ciudades ibéricas, los avances han sido también espectaculares. En el año 1954, García y Bellido describia en la ya citada Historia de España dirigida por $R$. Menéndez Pidal, el tipo de casa ibérica como «pobre y modesto, por lo general con un solo departamento habitable, a veces más, pero siempre ocupando el espacio estrictamente indispensable para vegetar al cobijo de la intemperie y conservar en ella el humilde patrimonio mueble de una familia: hasta el extremo de ser realmente pocas las veces en que el excavador halla, dentro de estos habitáculos, huellas diferenciadas de lo que en otras culturas primitivas representa el núcleo destacado de la familia y el eje principal de la casa: el hogar (...) Si la casa es de compartimentos múltiples, no se les adivina su destino especifico (...)m. Se deduce, pues, que la casa ibérica era realmente una casa primitiva y atrasada. aunque llamaba la atención acerca de que en algunas ciudades, como La Bastida y Azaila, se vislumbraban otras «que parecen estar formadas por dos o más compartimientos; pero es dificil aislarlos del conjunto abigarradơ de paredes vecinas y contiguas para reconstruir con ellos un posible conjunto domésticon; cree que, al menos en Azaila, ello pudo deberse ya a la influencia romana.

Para asentar su opinión acerca de las casas ibéricas, nuestro venerable maestro contó en realidad con bastantes pocos materiales: las casas de La Bastida Azaila, Calaceite, Puig Castellar y unas pocas más, esto es, con asentamientos en algunos casos ni siquiera ibéricos y todos ellos excavados en décadas 
anteriores, con los métodos y requerimientos del momento, que desperdiciaban buena parte de la información. Y como tal quedó durante bastantes años. En el libro ya citado, Antonio Arribas se lamenta de que la falta de excavaciones sistemáticas no haya permitido avanzar en el conocimiento de los poblados ibéricos. Durante mucho tiempo, esta situación se ha mantenido; hemos venido repitiendo tópicos acerca de la estructura urbana ibérica basándonos en los mismos yacimientos con que pudo contar García y Bellido, y en todo caso en alguno más, quizás mejor excavado pero sólo parcialmente publicado, como pudo ser Covalta, en los limites de Alicante y Valencia.

En las décadas siguientes, el conocimiento de la urbanística ibérica apenas prosperó. La más notable aportación fue la de E. Llobregat en su obra Contestania Ibérica, publicada en Alicante en 1972; el autor realizó una síntesis de todos los yacimientos conocidos $\mathrm{y}$, lo que es más importante desde nuestro punto de vista, llevó a cabo el primer intento de estructuración de las unidades de habitación del poblado de La Bastida, proponiendo, a partir del estudio de los materiales encontrados en los diferentes departamentos, que tal vez tuvieran relación con el lugar de habitación y de trabajo de un sexo concreto. Se iniciaba asi un nuevo camino en el planteamiento de los estudios de urbanismo ibérico. Otro, que tendrá que esperar años, es el estudio de la modulación de las diferentes estructuras, desarrollado en 1983 por F. Burillo en sus trabajos sobre El Taratrato y Los Castellones de Herrera de los Navarros. Y por último se incorporará el estudio de las técnicas constructivas ibéricas y de sus estructuras más peculiares; es la vía que recoge y sistematiza el libro de J. Maluquer Arquitecture i urbanisme ibèrics a Catalunya, publicado en Barcelona en 1986.

En los últimos años, se ha avanzado considerablemente en algunas de estas líneas de investigación, y muchas de ellas han sido objeto de un primer tratamiento en el coloquio Habitats etstructures domestiques en Méditerranée occidentale durant la Protohistoire celebrado en Arlés en 1989, cuya publicación constituirá sin duda un hito en este tipo de estudios.

Sin embargo, el conocimiento de la urbanística ibérica se encuentra constreñido por varias circunstancias dificilmente superables. En primer lugar, por la menor espectacularidad inmediata de los trabajos en ciudades y poblados con respecto a los de las necrópolis, que hace que se concentren con preferencia en estas últimas; en segundo lugar, por el hecho de que durante muchos años la mayor parte de los trabajos se hayan centrado en la realización de cortes estratigráficos y sólo muy raramente se hayan llevado a cabo actuaciones en extensión. Las primeras resultan sumamente útiles para conocer la fecha de los poblados y su mutua interrelación, pero para avanzar en su conocimiento se requiere el trabajo en amplias extensiones, que resultan caras y costosas para los recursos que actualmente se destinan a la Arqueologia.

La linea marcada por E. Llobregat fue seguida especialmente por los trabajos de H. Bonet, C. Mata, J. Bernabéu y P.Guérin, quienes en el Coloquio sobre el Microespacio de Teruel celebrado en 1986 analizaron los materiales encontrados en el Puntal dels Llops en Valencia, proponiendo un destino concreto a las habitaciones. J. Santos Velasco realizó por este mismo tiempo un ensayo de estudio espacial, diferenciando almacenes de zonas de habitación en La Bastida de Les Alcuses, el mismo yacimiento sobre el que trabajó E. Llobregat. Sin embargo, los nuevos trabajos llevados a cabo sobre este yacimiento por E. Dies y $\mathrm{H}$. Bonet, han puesto de manifiesto que la planta y las estructuras publicadas no se compadecen con la realidad, por lo que cualquier conclusión obtenida sobre éstas puede estar alejada de la realidad. En la actualidad se están realizando trabajos de este tipo en numerosos yacimientos: El oral, Puente Tablas, El Tossal de Sant Miquel en Liria, etc.

En el segundo aspecto, la modulación de las estructuras, el libro recientemente publicado por uno de los autores (L. Abad) y F. Sala sobre el poblado ibérico de El Oral, en San Fulgencio, Alicante, plantea la posibilidad de una cierta modulación, basada en el empleo de una unidad de medida de unos $36 \mathrm{~cm}$ y en la existencia de estructuras de dimensiones similares que se repiten en varios lugares del poblado.

$\mathrm{Y}$ en cuanto al tercero, los métodos más cuidadosos aplicados a las excavaciones en los últimos años han permitido encontrar aquellos hogares que Garcia y Bellido echaba de menos y documentar un amplisimo repertorio de hogares, desde los más simples a los más complejos; junto a ellos, bancos, umbrales, desagues, etc., han ido llenando poco a poco, aunque no tanto como quisiéramos, el vacío existente. El poblado de El oral, al que acabamos de referirnos algo más arriba, constituye un buen ejemplo.

De todo ello puede concluirse que las casas y las estructuras ibéricas son en realidad bastante más complejas de lo que se suponía en época de nuestro admirado maestro el profesor García y Bellido, en consonancia con la mayor complejidad que, como ha quedado claro a lo largo de esta Jornada, corresponde a la propia cultura ibérica. Pero para que podamos avanzar por este camino es necesario publicar todos los asentamientos que, de una u otra forma, 
se encuentran excavados; realizar trabajos en extensión en poblados y ciudades de diferentes tipos y en muchas áreas geográficas; intensificar las prospecciones, desde la conviceión de que deben ser completadas con el estudio de los principales asentamientos; estudiar la función de las casas dentro del asentamiento y de las habitaciones dentro de aquéllas, y la relación que pudo existir entre ciudades y necrópolis. Si somos capaces de llevar a cabo todo ello, el fenómeno urbano y el urbanismo en las culturas ibéricas, que ahora empiezan a entreverse. nos depararán en los próximos años sorpresas tan grandes como aquellas a las que hemos asistido desde el año 1954.

\title{
ESCULTURA IBÉRICA, AYER Y HOY: LA BICHA DE BALAZOTE
}

POR

\author{
MICHAEL BLECH \\ Instituto Arqueológico Alemán, Madrid
}

Las obras de A. Garcia y Bellido ', como la Hispania Graeca (1948) o Las esculturas romanas de la Peninsula Ibérica (1949), y sus diferentes apor-

\footnotetext{
' Se agradece la revisión del texto a $\mathrm{M}$. Tejeiro Díaz y algunas ideas a D. Marzoli y M. Luik Esta aportación presenta la versión resumida de mi charla "Escultura ibérica, ayer y hoy" dada por la amable invitación del Departamento de Arqueologia del CSIC. Mi aportación se concentra en una escultura, la famosa "Bicha de Balazote" tratada magistralmente por el homenajeado Antonio Garcia y Bellido en 1931.

Para la historia de la investigación sobre la escultura ibérica: Chapa Brunet 1980, 20-70; Chapa Brunet 1986; Ruano 1987. 14-53; cf. tambièn Almagro Basch, M.: Las raices del arte ibérico. Papeles del Laboratorio de Arqueologia de Valencia, 11,1975, 251-279. Para la bibliografia de la Bicha de Balazote véase Garcia y Bellido 1931; id. 1954, 578-580; Chapa 1980, 268 27I: id. 1981; id 1985, 234-240; id 1986, $121 \mathrm{n}^{\circ} 219,220-222$; Garcia-Bellido 1992, 83-92. Para el toro en las esculturas ibéricas véase Blanco, A .: El toro ibérico, en: Homenaje al prof. C. de Mergelina, Murcia 1961/62, 163-196; cf. además las notas 49-51.

Titulos abreviados: Almagro-Gorbea $=$ Almagro-Gorbea, $\mathrm{M}$. Pozo Moro, MadrMitt, 24, 1983, 177-293. Blech y Ruano 1992 = Blech, M. y Ruano Ruiz, E.: Zwei iberische Skulpturen aus Ubeda la Vieja (Jaén). MadrMitt 33, 1992, 70-111. Chapa Brunet $1980=$ Chapa Brunet, T.: La escultura zoomorfa ibérica en piedra, Madrid 1980. Chapa Brunet $1981=$ Chapa Brunet, T.: E toro androcéfalo de Balazote, Al-Basit 7, $\mathrm{n}^{\circ}$. 10, 1981, 145-157. Chapa Brunet $1985=$ Chapa Brunet, T.: La escultura ibérica zoomorfa, Madrid 1985. Chapa Brunet 1986a $=$ Chapa Brunet, T.: Escultura ibérica: Una revisión de sus interpretaciones, TrabPreHist, 43, 1986, 43-60. Chapa Brunet 1986b $=$ Chapa Brunet, T.: Influjos griegos en la escultura zoomorfa ibérica, Iberia Graeca, Ser. Arqueológica, 11, Madrid 1986. Chapa Brunet $1993=$ Chapa Brunet, T.: La destrucción de la escultura funeraria ibérica, TrabPrehist, 50, 1993, 183-185. Garcia y Bellido 1931a = Garcia y Bellido, A.: Las relaciones entre el arte etrusco e ibero, ArchEspArteA, 7, 1931, 119-148. García y Bellido $1931 \mathrm{~b}=$ García y
}

taciones al mundo ibérico ${ }^{2}$, abrieron vastos campos de conocimiento. Puntos de partida fueron sus cuidadosas descripciones de los objetos, la transfor-

Bellido, A.: La bicha de Balazote. ArchEspArteA, 7, 1931, 249270. García y Bellido $1943 \mathrm{a}=$ Garcia y Bellido, A.: La dama de Elche, Madrid, 1943. Garcia y Bellido 1943b = Garcia y Bellido, A.: Algunos problemas de arte y cronologia ibérica, ArchEspA. 16, 1943, 78-108. Garcia y Bellido $1943 \mathrm{c}=$ Garcia y Bellido, A: De escultura ibérica, algunos problemas de arte y cronologia, ArchEspA, 16, 1943, 272-299. Garcia y Bellido 1954=Garcia y Bellido, A.: Arte ibérico, en: Historia de España, Madrid 1954. vol. I 3, 371-675. Garcia y Bellido $1980=$ Garcia y Bellido, Arte ibérico en España. Madrid 1980. Garcia-Bellido $1990=$ Garcia-Bellido, M. P.: El tesoro de Mogente y su entorno monetal, Valencia 1990. Isler $1970=$ Isler, H. P.: Acheloos, Bern 1970. Isler 1981 = Isler, H. P.: Acheloos, en: L.IMC, Basel 1981, vol. I. 12-36. Jannot 1974 = Jannot, J. R.: Achéloos, le taureau androcéphale et les masques cornus dans l'Etrurie archaïque, Latomus, 33, 1974, 265-289. Llobregat $1966=$ Llobregat, E.A.: La escultura en piedra del Pais Valenciano. ArchArteValenciano, 37. 1966, 41-57. (Llobregat, E. A.: Ilucant 1991, 81-100). Llobregat 1972 = Llobregat, E. A.: Contestania ibérica, Alicante 1972. Llobregat $1991=$ Llobregat, E. A.: Ilucant, Alicante 1991. Olmos $1986=$ Olmos, R.: Anotaciones preliminares al libro de Teresa Chapa, en : Chapa 1986, 7-38. Olmos $1992 \mathrm{a}=$ Olmos, R.: El surgimiento de la imagen en la sociedad ibérica, en: Sociedad 1992, 8-32. Olmos $1992 \mathrm{~b}=$ Olmos, R.: Religiosidad y vida cotidiana en la España ibérica. Seminarios Fons Mellaria, 1991, 11-45. Paris 1903 = Paris, P.: Essai sur l'art et l'industrie de 1'Espagne primitive (1903). Ruano Ruiz 1987 = Ruano Ruiz, E.: La escultura humana de piedra en el mundo ibérico, vol 1-III (1987). Sociedad $1992 / 93=$ La sociedad ibérica a través de la imagen, Cat. Albacete etc.,1992/93. Tarradell $1965=$ Tarradell, M.: Arte ibérico, Barcelona 1968. Trillmich $1975=$ Trillmich, W.: Ein Kopffragment aus Verdolay bei Murcia, MadrMitt 16 , $1975,208-245$.

${ }^{2}$ Garcia y Bellido 193 la.b. 1943a.b. 1954. 1980. 